\title{
Correction to: Dual RNA-seq reveals viral infections in asthmatic children without respiratory illness which are associated with changes in the airway transcriptome
}

Agata Wesolowska-Andersen ${ }^{1}$, Jamie L. Everman ${ }^{1}$, Rebecca Davidson ${ }^{1}$, Cydney Rios ${ }^{1}$, Rachelle Herrin ${ }^{1}$, Celeste Eng' ${ }^{2}$, William J. Janssen ${ }^{3}$, Andrew H. Liu, ${ }^{4,5}$, Sam S. Oh², Rajesh Kumar ${ }^{6}$, Tasha E. Fingerlin 1,7, Jose Rodriguez-Santana ${ }^{8}$, Esteban G. Burchard ${ }^{2,9}$ and Max A. Seibold ${ }^{1,4,10^{*}}$

\section{Correction}

In our recent article [1], it has come to our attention that the sample labels are not consistent between Table 1, the data labels deposited in the Sequence Read Archive, and Additional file 1: Table S2. We are therefore providing an updated Additional file 1: Table S2 so identical samples now have the same label.
Published online: 10 April 2018

\section{Reference}

1. Wesolowska-Andersen A, Everman JL, Davidson R, Rios C, Herrin R, Eng C, Janssen WJ, Liu AH, Oh SS, Kumar R, et al. Dual RNA-seq reveals viral infections in asthmatic children without respiratory illness which are associated with changes in the airway transcriptome. Genome Biol. 2017;18:12.

\section{Additional file}

Additional file 1: Table S2. Gene count table for 48 RNA-seq nasal

brushing subjects from GALA cohort. (XLSX $5732 \mathrm{~kb}$ )

\begin{abstract}
Author details
'Center for Genes, Environment, and Health, National Jewish Health, Denver,

CO, USA. ${ }^{2}$ Department of Medicine, University of California, San Francisco,

CA, USA. ${ }^{3}$ Department of Medicine, National Jewish Health, Denver, CO, USA.

${ }^{4}$ Department of Pediatrics, National Jewish Health, 1400 Jackson St, Denver, CO 80206, USA. ${ }^{5}$ Children's Hospital Colorado and University of Colorado School of Medicine, Aurora, CO, USA. ${ }^{6}$ Department of Pediatrics, The Ann and Robert H. Lurie Children's Hospital of Chicago, Northwestern University Feinberg School of Medicine, Chicago, IL, USA. 'Department of Biomedical Research, National Jewish Health, Denver, CO, USA. ${ }^{8}$ Centro de Neumologia Pediatrica, San Juan, Puerto Rico. ${ }^{9}$ Department of Bioengineering and Therapeutic Sciences, University of California, San Francisco, CA, USA. ${ }^{10}$ Division of Pulmonary Sciences and Critical Care Medicine, University of Colorado School of Medicine, Aurora, CO, USA.
\end{abstract}

\section{* Correspondence: seiboldm@njhealth.org}

${ }^{1}$ Center for Genes, Environment, and Health, National Jewish Health, Denver, CO, USA

${ }^{4}$ Department of Pediatrics, National Jewish Health, 1400 Jackson St, Denver, CO 80206, USA 